Editora Chefe: Prof $^{\mathrm{a}} \mathrm{Dr}^{\mathrm{a}}$ Antonella Carvalho de Oliveira

Bibliotecário Maurício Amormino Júnior

Diagramação: Camila Alves de Cremo

Edição de Arte: Luiza Alves Batista

Revisão: Os Autores

Organizadores: ou Autores: Rafael Henrique Silva

Dados Internacionais de Catalogação na Publicação (CIP) (eDOC BRASIL, Belo Horizonte/MG)

158 Inovação e tecnologia para o cuidar em enfermagem 3 [recurso eletrônico] / Organizador Rafael Henrique Silva. - Ponta Grossa, PR: Atena, 2020.

Formato: PDF

Requisitos de sistema: Adobe Acrobat Reader. Modo de acesso: World Wide Web. Inclui bibliografia

ISBN 978-65-5706-310-1

DOI 10.22533/at.ed.101202108

1. Enfermagem - Pesquisa - Brasil. 2. Saúde Brasil. I. Silva, Rafael Henrique.

Elaborado por Maurício Amormino Júnior - CRB6/2422

Atena Editora

Ponta Grossa - Paraná - Brasil

Telefone: +55 (42) 3323-5493

www.atenaeditora.com.br contato@atenaeditora.com.br 


\section{SEGURANÇA DO PACIENTE EM ANGIOTOMOGRAFIA COMPUTADORIZADA: POR QUE O ACESSO VENOSO É IMPORTANTE?}

Data de aceite: 03/08/2020

Data de submissão: 06/05/2020

Beatriz Cavalcanti Juchem

Hospital de Clínicas de Porto Alegre Porto Alegre - RS http://lattes.cnpq.br/0923198465204339

\section{Alesandra Glaeser}

Hospital de Clínicas de Porto Alegre

Porto Alegre - RS http://lattes.cnpq.br/5160925545277815I

Jeane Cristine de Souza da Silveira Hospital de Clínicas de Porto Alegre Porto Alegre - RS http://lattes.cnpq.br/9507928549845245

Karine Bertoldi

Hospital de Clínicas de Porto Alegre Porto Alegre - RS http://lattes.cnpq.br/5919667702263498

Leticia Souza dos Santos Erig Hospital de Clínicas de Porto Alegre Porto Alegre - RS http://lattes.cnpq.br/4402548351260152

\section{Luciana Nabinger Menna Barreto}

Hospital de Clínicas de Porto Alegre

Porto Alegre - RS http://lattes.cnpq.br/4812391030682997

Sabrina Curia Johansson Timponi Hospital de Clínicas de Porto Alegre Porto Alegre - RS http://lattes.cnpq.br/5165037287911935
RESUMO: Nas últimas décadas, a angiotomografia computadorizada (angioTC) tem sido amplamente utilizada como uma ferramenta para o diagnóstico e tratamento de doenças vasculares. Fatores relacionados ao paciente, ao meio de contraste, assim como ao protocolo de injeção do contraste são determinantes para a qualidade do exame realizado. Entre os fatores descritos acima, a aquisição de um acesso venoso adequado para a realização do exame é fundamental. A obtenção de um acesso venoso adequado pode ser um desafio em pacientes que possuem uma rede venosa precária, assim como em pacientes pediátricos. Nestas situações, os acessos venosos centrais podem ser uma alternativa quando compatíveis com injeção por bomba injetora ("power-injectable"), desde que sejam respeitadas as instruções do fabricante para evitar eventos adversos, como ruptura ou deslocamento do cateter durante a injeção do contraste. O objetivo deste trabalho foi relatar a experiência de enfermeiras de um Serviço de Radiologia com a realização de angioTC, evidenciando a importância de um acesso venoso adequado para a realização deste exame e sua relação com a segurança do paciente.

PALAVRAS-CHAVE: Angiografia por Tomografia Computadorizada, Extravasamento de Materiais Terapêuticos e Diagnósticos, Meios de Contraste, Segurança do Paciente.

PATIENT SAFETY IN COMPUTED TOMOGRAPHY ANGIOGRAPHY: WHY IS VENOUS ACCESS IMPORTANT?

ABSTRACT: In the last decades, the computed 
tomography angiography (CTA) has been widely used as a tool for diagnosis and treatment of vascular diseases. Some factors related to the patient, the contrast medium and the contrast injection protocol are important to the exam quality. Among the factors described above, the acquisition of an adequate venous access for the examination is of great importance. This procedure can be challenging for patients with a precarious venous system as well as pediatric patients. In these situations, power-injection rated central venous catheters can be an alternative since the manufacturer's instructions are followed to avoid adverse events as catheter rupture or displacement during the contrast medium injection. The aim of this work was to describe the nurses' experience in a Radiology Service with the CTA procedure, demonstrating the importance of an adequate venous access to the exam and its relation with the patient safety.

KEYWORDS: Computed Tomography Angiography, Extravasation of Diagnostic and Therapeutic Materials, Contrast Media, Patient Safety.

\section{1 | INTRODUÇÃO}

A angiotomografia computadorizada (angioTC) surgiu na década de 90 e consiste em uma técnica de imagem minimamente invasiva, que possibilita o adequado mapeamento arterial e venoso, além de permitir uma visualização volumétrica do sistema vascular e anormalidades em grandes artérias sistêmicas e pulmonares. A partir do ano 2000, a angioTC tem superado a angiografia convencional como a modalidade preferida para 0 diagnóstico e caracterização da maioria das anomalias cardiovasculares, contribuindo também para o planejamento e avaliação do tratamento destas enfermidades (AMERICAN COLLEGE OF RADIOLOGY, 2016; FLEISCHMANN et al., 2016; REIS et al., 2015; RUBIN et al., 2014).

A técnica da angioTC consiste na aquisição de imagens enquanto o meio de contraste iodado $(\mathrm{MCl})$ proporciona o pico de realce nos vasos a serem estudados. Para isso, é necessária uma rápida injeção intravenosa do meio de contraste e um equipamento de tomografia de múltiplos detectores ou multislice (capaz de adquirir a imagem de várias "fatias" do corpo simultaneamente) que fará o tratamento do conjunto de dados com reconstruções multiplanares e renderizações em 3D (AMERICAN COLLEGE OF RADIOLOGY, 2016). Exemplos de imagens resultantes desta técnica são exibidas na Figura 1. 

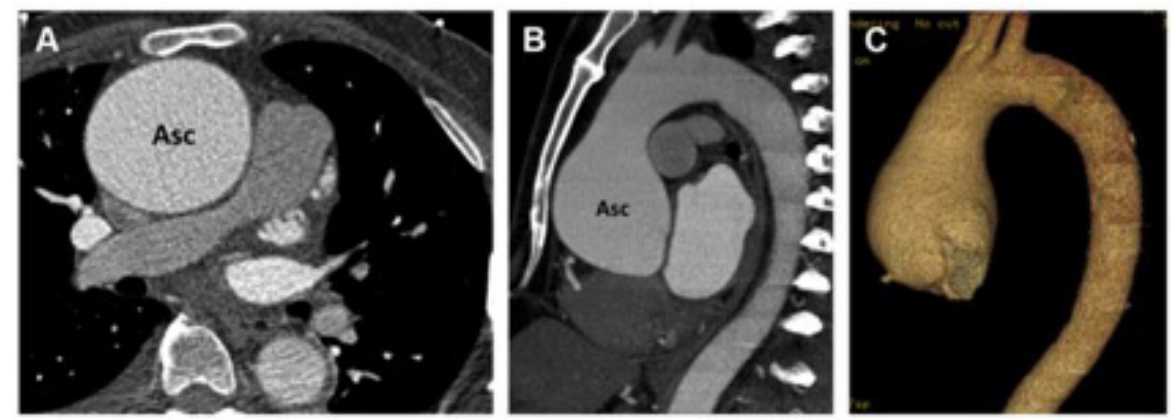

Figura 1 - Grande aneurisma da raiz da aorta e da aorta ascendente (Asc). A junção sinotubular é apagada e dilatada, criando a aparência clássica de bulbo de tulipa. Imagem em plano axial de aneurisma da aorta ascendente (A); imagem em plano coronal (B) e imagens tridimensionais renderizadas em volume (C) (SCHESKE, J. A. et al, 2016).

Diversos fatores podem influenciar diretamente na qualidade da imagem gerada e na precisão diagnóstica, podendo ser relacionados ao paciente (peso, débito cardíaco, volume de sangue circulante), ao $\mathrm{MCl}$ (concentração de iodo e viscosidade), assim como o protocolo de injeção do $\mathrm{MCl}$ (volume, velocidade e consequente duração da injeção) (FAGGIONI \& GABELLONI, 2016; FLEISCHMANN et al., 2016).

Em relação ao $\mathrm{MCl}$, normalmente é empregada uma solução não iônica a uma concentração de $350 \mathrm{mgl} / \mathrm{mL}$, pré aquecida em torno de $36^{\circ} \mathrm{C}$. É importante ressaltar que a dose deve ser calculada conforme o peso do paciente e a duração necessária da injeção, evitando doses excessivas que podem aumentar o risco de nefrotoxicidade (AMERICAN COLLEGE OF RADIOLOGY, 2016). Um exemplo de protocolo para pacientes com peso médio de $75 \mathrm{~kg}$ pode ser o seguinte (FLEISCHMANN et al., 2016):

- Tempo de aquisição da imagem: 10 segundos

- Duração da injeção: 18 segundos

- Velocidade da injeção: $5 \mathrm{ml} / \mathrm{seg}$ (necessário um volume de $90 \mathrm{ml}$ )

- Aquisição da imagem: iniciar automaticamente 8 segundos após a injeção de contraste.

Como citado acima, um dos fatores determinantes para a execução de um exame de qualidade depende da aquisição de imagens associada a uma rápida velocidade de injeção do meio de contraste (4 a $5 \mathrm{~mL} / \mathrm{s}$ ); portanto, a obtenção de um acesso venoso calibroso é fundamental para a realização do exame (AMERICAN COLLEGE OF RADIOLOGY, 2016; CAPUÑAY et al., 2009). Desta forma, este artigo tem o objetivo de relatar a experiência de enfermeiras de um Serviço de Radiologia com a realização de angioTC, evidenciando a importância de um acesso venoso adequado para a realização deste exame e sua relação com a segurança do paciente. 


\section{I MÉTODO}

Trata-se de um relato de experiência resultante da experiência clínica em tomografia de sete enfermeiras de um Serviço de Radiologia de um hospital universitário do sul do Brasil. Este estudo faz parte de um projeto intitulado: "Extravasamento de meio de contraste iodado em tomografia computadorizada: série de casos num hospital escola", aprovado pelo comitê de ética institucional sob o número 09-372.

\section{I RESULTADOS E DISCUSSÃO}

O acesso venoso considerado ideal para a realização de angioTC em pacientes adultos é aquele obtido na fossa antecubital, preferencialmente no membro superior direito, utilizando um dispositivo de calibre $20 \mathrm{G}$ ou $18 \mathrm{G}$ para punção periférica. Além disso, a permeabilidade do cateter deve ser previamente testada com a rápida injeção de um bolus de soro fisiológico (CAPUÑAY et al., 2009). Um cateter venoso central (CVC) compatível com injeção por bomba injetora ("power-injectable") também pode ser utilizado, desde que sejam respeitadas as instruções do fabricante (AMERICAN COLLEGE OF RADIOLOGY, 2016). Estes cateteres podem ser do tipo "power-PICC", "power-line" ou "power-port" para cateteres centrais de inserção periférica, cateteres mono ou multi-lumens ou cateteres implantados, respectivamente. Estes tipos de CVC, especificamente projetados para injeção automática do meio de contraste, podem suportar pressão de até 300 psi e fluxos de injeção do $\mathrm{MCl}$ de até $10 \mathrm{ml} / \mathrm{seg}$ (INDRAJIT, I. K. et al, 2015; KOLLI et al, 2018).

Devido à necessidade de injeção com alto fluxo, o extravasamento do $\mathrm{MCl}$ durante exames como a angioTC é um dos eventos adversos mais comuns. Além disso, um aumento no número de casos de extravasamento de $\mathrm{MCl}$ está relacionado com o uso generalizado de bombas injetoras (COMPAÑA et al., 2014). Os fatores de risco associados aos casos de extravasamento de $\mathrm{MCl}$ estão relacionados à técnica de injeção utilizada, assim como às condições do paciente. Em relação à técnica de injeção, podem ser citados fatores como o calibre do cateter, o local da punção, várias punções na mesma veia e a utilização de bomba injetora (pressão e velocidade do fluxo de injeção) (COMPAÑA et al., 2014). Pacientes idosos e crianças ou indivíduos com alteração do nível de consciência são mais suscetíveis, pois apresentam dificuldades em verbalizar a dor causada pelo extravasamento. Além disso, pacientes com tempo de internação prolongado (múltiplas punções), diversas comorbidades e em tratamentos como radioterapia e quimioterapia também são considerados mais suscetíveis. Quando existem muitos riscos para a realização de angioTC, há outra alternativa diagnóstica que consiste na angiorressonância magnética (angioRM). Este exame oferece outras avaliações estruturais concomitantes com técnicas de difusão e espectroscopia, além de permitir a obtenção das imagens sem o uso de meio de $\mathrm{MCl}$ e sem radiação ionizante (AMARAL, 2004). O meio de contraste utilizado na angioRM é o gadolínio, que tem menor incidência de reações adversas e complicações 
(JUCHEM et al, 2016). No entanto, esta modalidade diagnóstica está menos disponível do que a angioTC, demanda mais tempo para elucidação diagnóstica e exige a cooperação do paciente por tempo mais prolongado do que uma tomografia computadorizada (AMARAL, 2004). Desta forma, a angioTC ainda é mais utilizada como método diagnóstico justamente devido às vantagens de maior disponibilidade, menor custo e maior rapidez na execução do exame. No entanto, apesar do extravasamento ser um dos eventos adversos mais comuns, geralmente as consequências ao paciente são mínimas, como dor e edema no local, que regridem espontaneamente após algumas horas. Já os efeitos adversos graves são raros e estão relacionados a um maior volume extravasado (CAMPAÑA et al., 2014, NICOLA et al., 2016).

Para ilustrar a importância do que foi abordado até agora, vamos descrever brevemente o caso de paciente pediátrico que apresentou evento adverso relacionado ao acesso venoso durante exame de angioTC.

O Serviço de Radiologia admitiu um paciente masculino, de 8 anos, portador de síndrome do intestino curto, esteato-hepatite e diversos episódios de trombose venosa devido a mutação do fator $V$ de Leiden, que consiste em doença genética que favorece a hipercoabilidade (LEHMKUHL et al, 2012). Procedente do ambulatório, acompanhado pela mãe, vem realizar angioTC de região cervical, tórax, abdômen e membros superiores e inferiores para definição terapêutica e possível transplante hepático. Em uso de nutrição parenteral total domiciliar por CVC semi-implantado do tipo Broviac ${ }^{\circledR}$, inserido em veia femoral direita. A mãe e o paciente receberam orientações sobre a realização do exame e a mãe assinou o Termo de Consentimento autorizando o exame. Devido à precariedade da rede venosa periférica, o radiologista autorizou o uso do CVC para injeção do $\mathrm{MCl}$, apesar deste cateter não possuir a especificação "power-injectable". Durante o exame, o $\mathrm{MCl}$ foi administrado por bomba injetora a $2 \mathrm{ml} / \mathrm{seg}$, com pressão da injeção atingindo $70 \mathrm{psi} e$ alarme para interromper automaticamente a injeção caso a pressão chegasse ao limite de 325 psi. O paciente não apresentou queixas de dor ou desconforto durante a injeção. $\mathrm{Na}$ aquisição das imagens, observou-se coleção de $40 \mathrm{ml}$ de $\mathrm{MCl}$ na região abdominal, evidenciando extravasamento por rompimento do CVC (Figuras 2 e 3 ). 


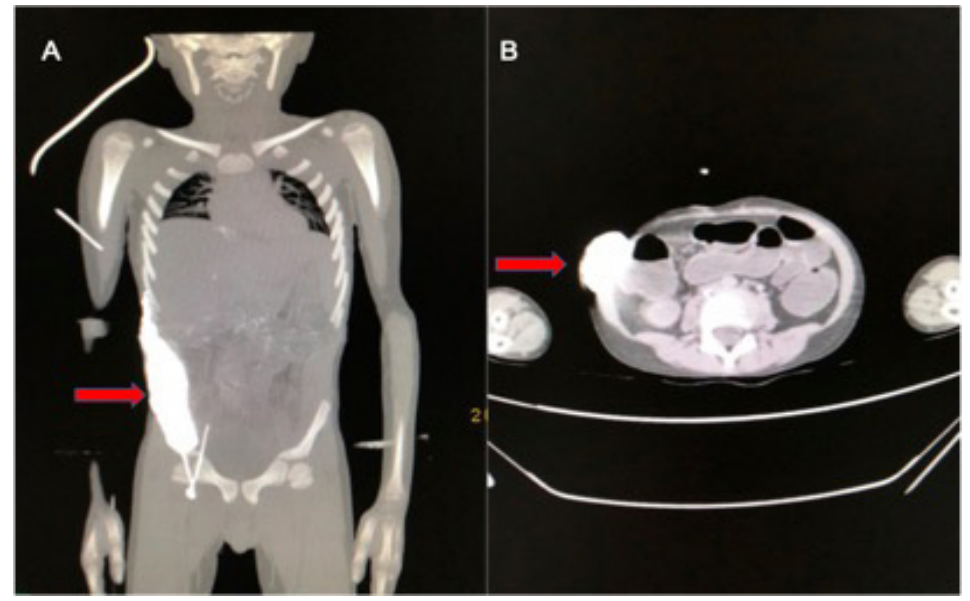

Figura 2 - Cortes coronal (A) e axial (B) demonstrando coleção do meio de contraste iodado na região subcutânea do hemiabdome $D$. Fonte: acervo das autoras.

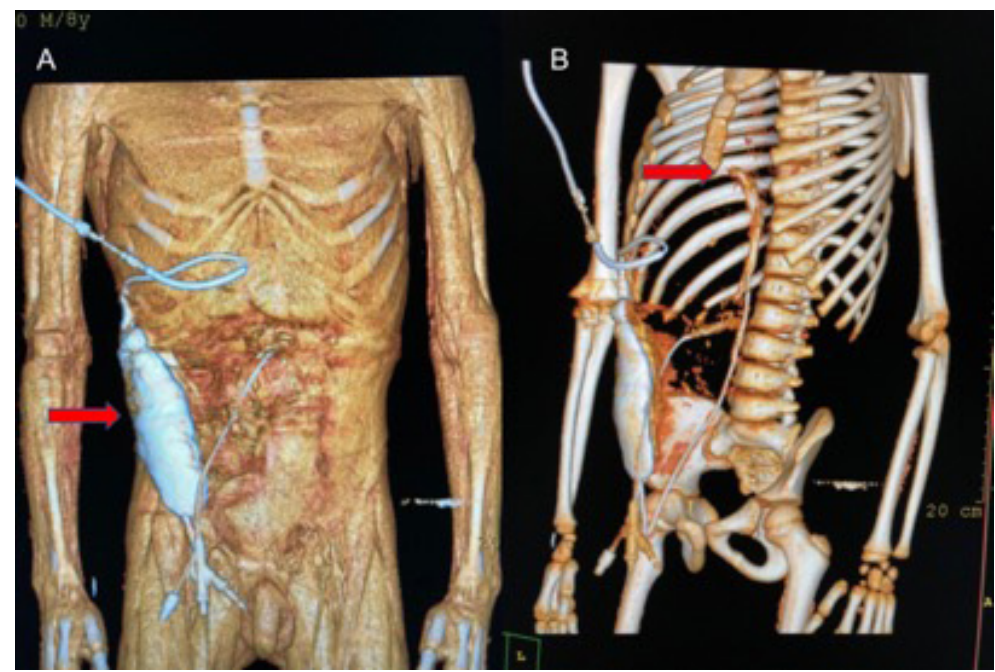

Figura 3 - Reconstruções em 3D demonstrando coleção do meio de contraste no hemiabdome $\mathrm{D}(\mathrm{A})$ e presença de trombo em veia cava $(\mathrm{B})$, impedindo a progressão do meio de contraste

O paciente apresentava abaulamento e leve hiperemia local, sem lesão de pele ou alteração dos sinais vitais e negava dor. Foi aplicado gelo na região afetada, havendo regressão parcial dos sintomas em uma hora. Posteriormente, o paciente foi encaminhado à internação pediátrica para acompanhamento e definição de conduta quanto ao CVC. Após 24 horas, o cateter foi trocado por novo Broviac ${ }^{\circledR}$ e uma ecografia abdominal evidenciou absorção completa do extravasamento, sem sequelas para o paciente, que recebeu alta hospitalar.

Em relação ao caso descrito acima, podemos discutir alguns fatores associados 
ao exame que podem ter ocasionado o evento adverso. A utilização do cateter Broviac ${ }^{\circledR}$ para a injeção do meio de contraste foi autorizada pelo radiologista responsável para realização do exame, devido à precariedade de rede venosa do paciente, assim como à urgência do exame para definição terapêutica. No entanto, é importante citar que, nas instruções do fabricante, havia uma contra-indicação para injeção de $\mathrm{MCl}$ utilizando bomba injetora. Portanto, mesmo tomando alguns cuidados prévios ao exame, como avaliação do posicionamento do cateter através de scout e testando a permeabilidade do cateter com soro fisiológico antes da injeção do $\mathrm{MCl}$, o Broviac ${ }^{\circledR}$ apresentou ruptura, provavelmente, por não resistir à pressão da injeção. Esse caso ilustra a importância de seguir as instruções do fabricante quanto ao uso de CVCs para injeção de meio de contraste.

Salienta-se que os CVCs de longa ou intermediária permanência (tunelizados, cateteres centrais de inserção periférica - PICC ou totalmente implantados como o portocath) tem sido frequente utilizados em pacientes pediátricos devido a necessidades terapêuticas e à precariedade de acesso venoso periférico (RIGSBY et al, 2007). Portanto, é importante que os serviços de radiologia estejam familiarizados com estes dispositivos e suas contraindicações, para evitar eventos adversos como este. Somente o acesso venoso periférico ou os cateteres compatíveis com infusão utilizando bomba injetora podem ser alternativas seguras para a realização de exames como a AngioTC, desde que as instruções do fabricante sejam estritamente seguidas.

\section{I CONSIDERAÇÕES FINAIS}

Um acesso venoso adequado para realização de angioTC é um fator fundamental para o sucesso do exame, assim como para a segurança do paciente. A familiaridade da equipe multiprofissional com os diferentes tipos de CVCs, suas especificidades e contraindicações é imprescindível para evitar eventos adversos relacionados ao seu uso.

A partir deste relato, sugere-se o desenvolvimento de outros estudos utilizando metodologias quantitativas, a fim de corroborar com a construção do conhecimento nesta área de atuação.

\section{REFERÊNCIAS}

AMARAL, Leonardo Portugal Guimarães; DOS SANTOS, Alair Augusto SMD; MARCHIORI, Edson.

Angiorressonância magnética do crânio: revisão de 100 casos. Radiologia Brasileira, v. 37, n. 3, p. 153-158, 2004.

AMERICAN COLLEGE OF RADIOLOGY. ACR-NASCI-SIR-SPR Practice parameter for the performance and interpretation of body Computed Tomography Angiography (CTA). ACR American College of Radiology. https://www.acr.org/-/media/ACR/Files/Practice-Parameters/Body-CTA. pdf?la=en. Published 2011. Updated 2016. Accessed 1 May. 
COMPAÑA, FJ Pacheco; VIDAL, B. Gago; DÍAZ, C. Méndez. Extravasation of contrast media at the puncture site: Strategies for management. Radiología (English Edition), v. 56, n. 4, p. 295-302, 2014.

CAPUÑAY, Carlos et al. Multidetector CT angiography and virtual angioscopy of the abdomen. Abdominal imaging, v. 34, n. 1, p. 81, 2009.

FAGGIONI, Lorenzo; GABELLONI, Michela. lodine concentration and optimization in computed tomography angiography: current issues. Investigative radiology, v. 51, n. 12, p. 816-822, 2016.

FLEISCHMANN, Dominik et al. Computed tomography angiography: a review and technical update. Radiologic Clinics, v. 54, n. 1, p. 1-12, 2016.

INDRAJIT, Inna K. et al. Pressure injectors for radiologists: A review and what is new. The Indian journal of radiology \& imaging, v. 25, n. 1, p. 2, 2015.

JUCHEM, Beatriz Cavalcanti et al. Embolia pulmonar. In: Margarita Ana Rubin Unicovsky; Beatriz Ferreira Waldman; Renê dos Santos Spezani. (Org.). PROENF - Urgência e Emergência - Ciclo 3. 1ed. Porto Alegre: Artmed Panamericana, v. 4, p. 11-40, 2016.

KOLLI, P. et al. Intravenous CT; X-ray Contrast Guidelines - Vascular Access and Use of Central Lines and Ports in AdultsSanFranciscoUCSF Radiology, , 2018. Disponível em: <https://radiology. ucsf.edu/patient-care/patient-safety/contrast/iodinated>. Acesso em: 2 maio. 2020

LEHMKUHL, Rafaela Ludwig et al. Mutação do fator $\mathbf{V}$ de Leiden em paciente com síndrome de Down. Relato de caso. Revista da Sociedade Brasileira de, v. 10, n. 6, p. 554-6, 2012.

NICOLA, Refky et al. Contrast media extravasation of computed tomography and magnetic resonance imaging: management guidelines for the radiologist. Current problems in diagnostic radiology, v. 45, n. 3, p. 161-164, 2016.

RIGSBY, Cynthia K. et al. Safety and efficacy of pressure-limited power injection of iodinated contrast medium through central lines in children. American Journal of Roentgenology, v. 188, n. 3 , p. 726-732, 2007.

RUBIN, Geoffrey D. et al. CT angiography after 20 years: a transformation in cardiovascular disease characterization continues to advance. Radiology, v. 271, n. 3, p. 633-652, 2014.

REIS, Fatima Regina Silva; CARDIA, Patricia Prando; D'IPPOLITO, Giuseppe. Computed tomography angiography in patients with active gastrointestinal bleeding. Radiologia brasileira, $v$. 48, n. 6, p. 381-390, 2015.

SCHESKE, Jonathan A. et al. Computed tomography angiography of the thoracic aorta. Radiologic Clinics, v. 54, n. 1, p. 13-33, 2016. 